\title{
Projective modules over overrings of polynomial rings and a question of Quillen
}

\author{
Manoj K. Keshari and Swapnil A. Lokhande 1
}

\begin{abstract}
Let $(R, \mathfrak{m}, K)$ be a regular local ring containing a field $k$ such that either char $k=0$ or char $k=p$ and tr-deg $K / \mathbb{F}_{p} \geq 1$. Let $g_{1}, \ldots, g_{t}$ be regular parameters of $R$ which are linearly independent modulo $\mathfrak{m}^{2}$. Let $A=R_{g_{1} \cdots g_{t}}\left[Y_{1}, \ldots, Y_{m}, f_{1}\left(l_{1}\right)^{-1}, \ldots, f_{n}\left(l_{n}\right)^{-1}\right]$, where $f_{i}(T) \in k[T]$ and $l_{i}=a_{i 1} Y_{1}+\ldots+a_{i m} Y_{m}$ with $\left(a_{i 1}, \ldots, a_{i m}\right) \in k^{m}-(0)$. Then every projective $A$-module of rank $\geq t$ is free. Laurent polynomial case $f_{i}\left(l_{i}\right)=Y_{i}$ of this result is due to Popescu.
\end{abstract}

\section{Introduction}

In this paper, we will assume that rings are commutative Noetherian, modules are finitely generated, projective modules are of constant rank and $k$ will denote a field.

Let $R$ be a ring and $P$ a projective $R$-module. We say that $P$ is cancellative if $P \oplus R^{m} \stackrel{\sim}{\rightarrow} Q \oplus R^{m}$ for some projective $R$-module $Q$ implies $P \stackrel{\sim}{\rightarrow} Q$. For simplicity of notations, we begin with a definition.

Definition 1.1 A ring $A=R\left[Y_{1}, \ldots, Y_{m}, f_{1}\left(l_{1}\right)^{-1}, \ldots, f_{n}\left(l_{n}\right)^{-1}\right]$ is said to be of type $R[d, m, n]$ if $R$ is a ring of dimension $d, Y_{1}, \ldots, Y_{m}$ are variables over $R$, each $f_{i}(T) \in R[T]$ and either each $l_{i}=Y_{i_{j}}$ for some $i_{j}$, or $R$ contains a field $k$ and $l_{i}=\sum_{j=1}^{m} a_{i j} Y_{j}-b_{i}$ with $b_{i} \in R$ and $\left(a_{i 1}, \ldots, a_{i m}\right) \in k^{m}-(0)$.

Let $A$ be a ring of the type $R[d, m, n]$. We say that $A$ is of type $R[d, m, n]^{*}$ if $f_{i}(T) \in k[T]$ and $b_{i} \in k$ for all $i$.

Let $A=R\left[Y_{1}, \ldots, Y_{m}, f_{1}\left(Y_{1}\right)^{-1}, \ldots, f_{n}\left(Y_{n}\right)^{-1}\right]$ be a ring of type $R[d, m, n]$ with $n \leq m$ and $l_{i}=Y_{i}$. If $P$ is a projective $A$-module of $\operatorname{rank} \geq \max \{2, d+1\}$, then Dhorajia-Keshari ([5], Theorem 3.12), proved that $E(A \oplus P)$ acts transitively on $\operatorname{Um}(A \oplus P)$ and hence $P$ is cancellative. This result was proved by Bass [2] in case $n=m=0$; Plumstead [12] in case $m=1, n=0$; Rao [16] in case $n=0$; Lindel [8] in case $f_{i}=Y_{i}$. Gabber [6] proved the following result: Let $k$ be a field and $A$ a ring of type $k[0, m, n]$. Then every projective $A$-module is free. We prove the following result (3.4) which generalizes ([5], Theorem 3.12) and is motivated by Gabber's result.

Theorem 1.2 Let $A=R\left[Y_{1}, \ldots, Y_{m}, f_{1}\left(l_{1}\right)^{-1}, \ldots, f_{n}\left(l_{n}\right)^{-1}\right]$ be a ring of type $R[d, m, n]$ and $P$ a projective $A$-module of $\operatorname{rank} \geq \max \{2, d+1\}$. Then $E(A \oplus P)$ acts transitively on $\operatorname{Um}(A \oplus P)$. In particular, $P$ is cancellative.

\footnotetext{
${ }^{1}$ Department of Mathematics, IIT Bombay, Mumbai - 400076, India; (keshari,swapnil)@math.iitb.ac.in
} 
The Bass-Quillen conjecture [3, 15] says: If $R$ is a regular ring, then every projective module over $R\left[X_{1}, \ldots, X_{r}\right]$ is extended from $R$. In $\mathrm{B}-\mathrm{Q}$ conjecture, we may assume that $R$ is a regular local ring, due to Quillen's local-global principal [15]: For a ring B, projective module $P$ over $B\left[X_{1}, \ldots, X_{r}\right]$ is extended from $B$ if and only if $P_{\mathfrak{m}}$ is free for every maximal ideal $\mathfrak{m}$ of $B$. We remark that Quillen's local global principal is also true for projective modules over positive graded rings ([19], Theorem 3.1), whereas it is not true for Laurent polynomial rings (44, Example 2, p. 809).

Lindel 9 gave an affirmative answer to B-Q conjecture when $R$ is a regular $k$-spot, i.e. $R=R_{\mathfrak{p}}^{\prime}$, where $R^{\prime}$ is some affine $k$-algebra and $\mathfrak{p}$ is a regular prime ideal of $R^{\prime}$. Using Lindel's result, Popescu [13. proved B-Q conjecture when $R$ is any regular local ring containing a field $k$.

Let $(R, \mathfrak{m})$ be a regular local ring. We say that $f \in \mathfrak{m}$ is a regular parameter of $R$ if $f$ is part of a minimal generating set of $\mathfrak{m}$. This is equivalent to $f \in \mathfrak{m}-\mathfrak{m}^{2}$. Further, let $g_{1}, \ldots, g_{t} \in \mathfrak{m}$ be regular parameters. Then $g_{1}, \ldots, g_{t}$ are linearly independent modulo $\mathfrak{m}^{2}$ if and only if $g_{1}, \ldots, g_{t}$ are part of a minimal generating set of $\mathfrak{m}$.

Quillen [15] had asked the following question whose affirmative answer would imply that B-Q conjecture is true: Assume $(R, \mathfrak{m})$ is a regular local ring and $f \in \mathfrak{m}$ a regular parameter of $R$. Is every projective $R_{f}$-module free?

Bhatwadekar-Rao 4 answered Quillen's question when $R$ is a regular $k$-spot. More generally, they proved: Let $(R, \mathfrak{m})$ be a regular $k$-spot with infinite residue field and $f$ a regular parameter of $R$. If $B$ is one of $R, R(T)$ or $R_{f}$, then projective modules over $B\left[X_{1}, \ldots, X_{r}, Y_{1}^{ \pm 1}, \ldots, Y_{s}^{ \pm 1}\right]$ are free.

Rao 17] generalized above result as follows: Let $(R, \mathfrak{m})$ be a regular $k$-spot with infinite residue field. Let $g_{1}, \ldots, g_{t}$ be regular parameters of $R$ which are linearly independent modulo $\mathfrak{m}^{2}$. If $A=R_{g_{1} \ldots g_{t}}\left[X_{1}, \ldots, X_{r}, Y_{1}^{ \pm 1}, \ldots, Y_{s}^{ \pm 1}\right]$, then projective $A$-modules of rank $\geq \min \{t, d / 2\}$ are free.

Popescu [14] generalized Rao's result as follows: Let $(R, \mathfrak{m}, K)$ be a regular local ring containing a field $k$ such that either char $k=0$ or char $k=p$ and $\operatorname{tr}$-deg $K / \mathbb{F}_{p} \geq 1$. Let $g_{1}, \ldots, g_{t}$ be regular $p a-$ rameters of $R$ which are linearly independent modulo $\mathfrak{m}^{2}$. If $A=R_{g_{1} \ldots g_{t}}\left[X_{1}, \ldots, X_{r}, Y_{1}^{ \pm 1}, \ldots, Y_{s}^{ \pm 1}\right]$, then projective $A$-modules of rank $\geq t$ are free.

We generalize Popescu's result as follows (5.8):

Theorem 1.3 Let $(R, \mathfrak{m}, K)$ be a regular local ring containing a field $k$ such that either char $k=0$ or char $k=p$ and tr-deg $K / \mathbb{F}_{p} \geq 1$. Let $g_{1}, \ldots, g_{t}$ be regular parameters of $R$ which are linearly independent modulo $\mathfrak{m}^{2}$. If $A=R_{g_{1} \ldots g_{t}}\left[Y_{1}, \ldots, Y_{m}, f_{1}\left(l_{1}\right)^{-1}, \ldots, f_{n}\left(l_{n}\right)^{-1}\right]$ is a ring of type $R_{g_{1} \ldots g_{t}}[d-1, m, n]^{*}$, then every projective $A$-module of rank $\geq t$ is free.

Note that we can not expect (1.3) for rings of type $R[d, m, n]$. For example, let $R$ be either $\mathbb{R}[X, Y]_{(X, Y)}$ or $\mathbb{R}[[X, Y]]$ and $A=R\left[Z, f(Z)^{-1}\right]$ a ring of type $R[2,1,1]$, where $f(T)=T^{2}+X^{2}+$ $Y^{2}$. Then stably free $A$-module $P$ of rank 2 given by the kernel of the surjection $(X, Y, Z): A^{3} \rightarrow A$ is not free. This will follow from the fact that $P$ over the rings $\mathbb{R}[X, Y, Z]_{(X, Y, Z)}\left[f(Z)^{-1}\right]$ or $\mathbb{R}[[X, Y, Z]]\left[f(Z)^{-1}\right]$ is not free (4, p. 808) and ([11, p. 366). 


\section{Preliminaries}

Let $A$ be a ring and $M$ an $A$-module. We say $m \in M$ is unimodular if there exist $\phi \in M^{*}=$ $\operatorname{Hom}_{A}(M, A)$ such that $\phi(m)=1$. The set of all unimodular elements of $M$ is denoted by $\operatorname{Um}(M)$. For an ideal $J \subset A$, we denote by $E^{1}(A \oplus M, J)$, the subgroup of $A u t_{A}(A \oplus M)$ generated by all the automorphisms

$$
\Delta_{a \varphi}=\left(\begin{array}{cc}
1 & a \varphi \\
0 & i d_{M}
\end{array}\right) \quad \text { and } \quad \Gamma_{m}=\left(\begin{array}{cc}
1 & 0 \\
m & i d_{M}
\end{array}\right)
$$

with $a \in J, \varphi \in M^{*}$ and $m \in M$. In particular, if $E_{r+1}(A)$ is the group generated by elementary matrices over $A$, then $E_{r+1}^{1}(A, J)$ denotes the subgroup of $E_{r+1}(A)$ generated by

$$
\Delta_{\mathbf{a}}=\left(\begin{array}{cc}
1 & \mathbf{a} \\
0 & i d_{F}
\end{array}\right) \quad \text { and } \quad \Gamma_{\mathbf{b}}=\left(\begin{array}{cc}
1 & 0 \\
\mathbf{b}^{t} & i d_{F}
\end{array}\right)
$$

where $F=A^{r}, \mathbf{a} \in J F$ and $\mathbf{b} \in F$. We write $E^{1}(A \oplus M)$ for $E^{1}(A \oplus M, A)$.

By $\operatorname{Um}^{1}(A \oplus M, J)$, we denote the set of all $(a, m) \in \operatorname{Um}(A \oplus M)$ with $a \in 1+J$, and $\operatorname{Um}(A \oplus M, J)$ denotes the set of all $(a, m) \in \mathrm{Um}^{1}(A \oplus M)$ with $m \in J M$. We write $\operatorname{Um}_{r}(A, J)$ for $\operatorname{Um}\left(A \oplus A^{r-1}, J\right)$ and $\mathrm{Um}_{r}^{1}(A, J)$ for $\mathrm{Um}^{1}\left(A \oplus A^{r-1}, J\right)$.

Let $p \in M$ and $\varphi \in M^{*}$ be such that $\varphi(p)=0$. Let $\varphi_{p} \in \operatorname{End}(M)$ be defined as $\varphi_{p}(q)=\varphi(q) p$. Then $1+\varphi_{p}$ is a (unipotent) automorphism of $M$. An automorphism of $M$ of the form $1+\varphi_{p}$ is called a transvection of $M$ if either $p \in \operatorname{Um}(M)$ or $\varphi \in \operatorname{Um}\left(M^{*}\right)$. We denote by $E(M)$, the subgroup of $\operatorname{Aut}(M)$ generated by all transvections of $M$.

The following result is due to Bak-Basu-Rao ([1], Theorem 3.10). In [5], we proved results for $E^{1}(A \oplus P)$. Due to this result, we can interchange $E(A \oplus P)$ and $E^{1}(A \oplus P)$.

Theorem 2.1 Let $A$ be a ring and $P$ a projective $A$-module of rank $\geq 2$. Then $E^{1}(A \oplus P)=$ $E(A \oplus P)$.

The following result follows from the definition.

Lemma 2.2 Let $I \subset J$ be ideals of a ring $A$ and $P$ a projective $A$-module. Then the natural map $E^{1}(A \oplus P, J) \rightarrow E^{1}\left(\frac{A}{I} \oplus \frac{P}{I P}, \frac{J}{I}\right)$ is surjective.

The following result is due to Gabber ([], Theorem 2.1).

Theorem 2.3 Let $k$ be a field and $A=k\left[Y_{1}, \ldots, Y_{m}, f_{1}\left(l_{1}\right)^{-1}, \ldots, f_{n}\left(l_{n}\right)^{-1}\right]$ a ring of type $k[0, m, n]$. Then every projective A-module is free.

Recall that a ring $R$ is essentially of finite type over a ring $B$ if $R$ is localization of an affine $B$-algebra $C$ at some multiplicative closed subset of $C$. The following result is due to Popescu ([13, Theorem 3.1). 
Theorem 2.4 Let $R$ be a regular local ring containing a field. Then $R$ is a filtered inductive limit of regular local rings essentially of finite type over $\mathbb{Z}$.

The following result is due to Wiemers ([20, Proposition 2.5).

Proposition 2.5 Let $R$ be a ring of dimension $d$ and $A=R\left[X_{1}, \ldots, X_{r}, Y_{1}^{ \pm 1}, \ldots, Y_{s}^{ \pm 1}\right]$. Let $c \in\left\{1, X_{r}, Y_{s}-1\right\}$. If $s \in R$ and $r \geq \max \{3, d+2\}$, then $E_{r}^{1}(A, s c A)$ acts transitively on $\mathrm{Um}_{r}^{1}(A, s c A)$.

The following result is due to Lindel ([8], Lemma 1.1).

Lemma 2.6 Let $A$ be a ring and $P$ a projective $A$-module of rank $r$. Then there exist $s \in A$, $p_{1}, \ldots, p_{r} \in P$ and $\phi_{1}, \ldots, \phi_{r} \in \operatorname{Hom}(P, A)$ such that following holds: $P_{s}$ is free, $\left(\phi_{i}\left(p_{j}\right)\right)=$ diagonal $(s, \ldots, s), s P \subset p_{1} A+\ldots+p_{r} A$, the image of $s$ in $A_{r e d}$ is a non-zerodivisor and $(0$ : $s A)=\left(0: s^{2} A\right)$.

Definition 2.7 Let $R \subset S$ be rings and $h \in R$ be a non-zerodivisor in $R$ and $S$ both. If the natural map $R / h R \rightarrow S / h S$ is an isomorphism, then we say $R \rightarrow S$ is an analytic isomorphism along $h$. In this case, we get the following fiber product diagram

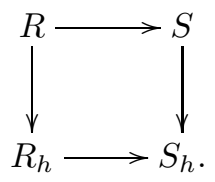

In particular, if $P$ is a projective $S$-module such that $P_{h}$ is free, then $P$ is extended from $R$.

The following result is due to Nashier ([10], Theorem 2.8). See also (44, Proposition, p. 803).

Proposition 2.8 Let $(R, \mathfrak{m})$ be a regular $k$-spot over a perfect field $k$. Let $g \in \mathfrak{m}$ and $f$ be any regular parameter of $R$ with $(g, f)$ a regular sequence. Then there exist a field $K / k$ and a regular $K$-spot $R^{\prime}$ such that

(i) $R^{\prime}=K\left[Z_{1}, \ldots, Z_{d}\right]_{\left(\phi\left(Z_{1}\right), Z_{2}, \ldots, Z_{d}\right)}$, where $\phi\left(Z_{1}\right) \in K\left[Z_{1}\right]$ is an irreducible monic polynomial. Moreover, we may assume $Z_{d}=f$.

(ii) $R^{\prime} \subset R$ is an analytic isomorphism along $h$ for some $h \in g R \cap R^{\prime}$.

(iii) If $R / \mathfrak{m}$ is infinite, then $K$ is also infinite.

We state a result due to Keshari ([7, Lemma 3.3).

Lemma 2.9 Let $A$ be a ring and $P$ a projective A-module of rank $r$. Choose $s \in A$ satisfying the properties of (2.6). Assume that $R^{r}$ is cancellative, where $R=A[X] /\left(X^{2}-s^{2} X\right)$. Then every element of $\mathrm{Um}^{1}\left(A \oplus P, s^{2} A\right)$ can be taken to $(1,0)$ by some element of $A u t(A \oplus P)$.

We end this section with following result of Bhatwadekar-Rao (4], Proposition 3.7).

Proposition 2.10 Let $B$ be a reduced ring of dimension $d$ and $R$ an overring of $B[X]$ contained in its total quotient ring. Let $A=R\left[X_{1}, \ldots, X_{n}, Y_{1}^{ \pm 1}, \ldots, Y_{m}^{ \pm 1}\right]$. Then $A^{r}$ is cancellative for $r \geq d+1$. 


\section{Cancellation over overrings of polynomial rings}

In this section, we prove our first result (3.4). We begin with the following:

Proposition 3.1 Let $A=R\left[Y_{1}, \ldots, Y_{m}, f_{1}\left(l_{1}\right)^{-1}, \ldots, f_{n}\left(l_{n}\right)^{-1}\right]$ be a ring of type $R[d, m, n]$. If $s \in R$ and $r \geq \max \{3, d+2\}$, then $E_{r}^{1}(A, s A)$ acts transitively on $\operatorname{Um}_{r}^{1}(A, s A)$.

Proof By ([5], Lemma 3.1), we may assume that $R$ is reduced. The case $n=0$ follows from (2.5). Assume $n>0$ and use induction on $n$. The case each $l_{j}=Y_{i_{j}}$ is proved in ([5], Proposition 3.5). We will prove the other case.

Let $\left(a_{1}, \ldots, a_{r}\right) \in \operatorname{Um}_{r}^{1}(A, s A)$. Recall that $l_{n}=a_{n 1} Y_{1}+\ldots+a_{n m} Y_{m}-b_{n}$ with $\left(a_{n 1}, \ldots, a_{n m}\right) \in$ $k^{m}-(0)$ and $b_{n} \in R$. We can find $\theta \in E_{m}(k)$ such that $\theta\left(a_{n 1}, \ldots, a_{n m}\right)=(0, \ldots, 0,1)$. Replacing the variables $\left(Y_{1}, \ldots, Y_{m}\right)$ by $\theta\left(Y_{1}, \ldots, Y_{m}\right)$, we may assume that $l_{n}=Y_{m}-b_{n}$. Further replacing $Y_{m}$ by $Y_{m}+b_{n}$, we may assume that $l_{n}=Y_{m}$.

Let $S=1+f_{n}\left(Y_{m}\right) R\left[Y_{m}\right]$. Then $A_{S}=B\left[Y_{1}, \ldots, Y_{m-1}, f_{1}\left(l_{1}\right)^{-1}, \ldots, f_{n-1}\left(l_{n-1}\right)^{-1}\right]$, where $B=R\left[Y_{m}\right]_{f_{n}\left(Y_{m}\right) S}$ is a ring of dimension $d, l_{i}=\sum_{j=1}^{m-1} a_{i j} Y_{j}+\widetilde{b}_{i}$ with $\widetilde{b}_{i}=a_{i m} Y_{m}-b_{i} \in B$. Hence $A_{S}$ is of type $B[d, m-1, n-1]$. By induction on $n$, we can find $\sigma \in E_{r}^{1}\left(A_{S}, s A_{S}\right)$ such that $\sigma\left(a_{1}, \ldots, a_{r}\right)=(1,0, \ldots, 0)$. We can find $g=1+f_{n}\left(Y_{m}\right) h\left(Y_{m}\right) \in S$ and $\sigma^{\prime} \in E_{r}^{1}\left(A_{g}, s A_{g}\right)$ such that $\sigma^{\prime}\left(a_{1}, \ldots, a_{r}\right)=(1,0, \ldots, 0)$. Rest of the proof is similar to ([5], Proposition 3.5). Hence we only give a sketch.

Let $C=R\left[Y_{1}, \ldots, Y_{m}, f_{1}\left(l_{1}\right)^{-1}, \ldots, f_{n-1}\left(l_{n-1}\right)^{-1}\right]$ be a ring of type $R[d, m, n-1]$. Consider the fiber product diagram

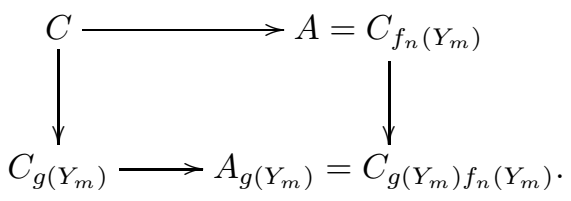

By ([5], Lemma 3.2), there exist $\sigma_{1} \in E_{r}^{1}\left(C_{f_{n}}, s\right)$ and $\sigma_{2} \in \mathrm{SL}_{r}^{1}\left(C_{g}, s\right)$ such that $\sigma^{\prime}$ has a splitting $\sigma^{\prime}=\left(\sigma_{2}\right)_{f_{n}} \circ\left(\sigma_{1}\right)_{g}$. Patching unimodular elements $\sigma_{1}\left(a_{1}, \ldots, a_{r}\right) \in \operatorname{Um}_{r}^{1}\left(C_{f_{n}}, s\right)$ and $\left(\sigma_{2}\right)^{-1}(1,0, \ldots, 0) \in \operatorname{Um}_{r}^{1}\left(C_{g}, s\right)$, we get $\left(c_{1}, \ldots, c_{r}\right) \in \operatorname{Um}_{r}^{1}(C, s)$. By induction on $n$, there exist $\phi \in E_{r}^{1}(C, s)$ such that $\phi\left(c_{1}, \ldots, c_{r}\right)=(1,0, \ldots, 0)$. Taking projection of $\phi$ in $A$, we get $\Phi \in E_{r}^{1}(A, s)$ such that $\Phi \sigma_{1}\left(a_{1}, \ldots, a_{r}\right)=(1,0, \ldots, 0)$. This completes the proof.

As a consequence of (3.1), we get the following:

Proposition 3.2 Let $A=R\left[Y_{1}, \ldots, Y_{m}, f_{1}\left(l_{1}\right)^{-1}, \ldots, f_{n}\left(l_{n}\right)^{-1}\right]$ be a ring of type $R[d, m, n]$. Then

(i) the canonical map $\Phi_{r}: \mathrm{GL}_{r}(A) / E_{r}(A) \rightarrow K_{1}(A)$ is surjective for $r \geq \max \{2, d+1\}$.

(ii) Further assume $f_{i}(T) \in R[T]$ is monic polynomial, $n \leq m$ and $l_{i} \in k\left[Y_{1}, \ldots, Y_{i}\right]$ with $a_{i i} \neq 0$ (see 1.1). Then for $r \geq \max \{3, d+2\}$, any stably elementary matrix in $\mathrm{GL}_{r}(A)$ is in $E_{r}(A)$. In particular, $\Phi_{d+2}$ is an isomorphism. 
Proof The proof of $(i)$ is same as ([5], Theorem 3.8). For $(i i)$, let $M \in \mathrm{GL}_{r}(A)$ be a stably elementary matrix. In case $n=0$ or each $l_{i}=Y_{i}$, the proof follows from ([5], Theorem 3.8). Assume $n>0$ and use induction on $n$. Recall that $l_{n}=a_{n 1} Y_{1}+\ldots+a_{n n} Y_{n}-b_{n}$ with $a_{n n} \neq 0$. Changing $Y_{n} \mapsto$ $a_{n n}^{-1}\left(Y_{n}-a_{n 1} Y_{1}-\ldots-a_{n-1, n-1} Y_{n-1}\right)+b_{n}$, we may assume that $l_{n}=Y_{n}$. Let $S=1+f_{n}\left(Y_{n}\right) R\left[Y_{n}\right]$ and $B=R\left[Y_{n}\right]_{f_{n} S}$. Then $A_{S}=B\left[Y_{1}, \ldots, Y_{n-1}, Y_{n+1}, \ldots, Y_{m}, f_{1}\left(l_{1}\right)^{-1}, \ldots, f_{n-1}\left(l_{n-1}\right)^{-1}\right]$ is a ring of type $B[d, m-1, n-1]$ with $l_{i} \in k\left[Y_{1}, \ldots, Y_{i}\right]$ and $a_{i i} \neq 0$. By induction on $n, M_{S} \in E_{r}\left(A_{S}\right)$. Hence we can choose $g \in S$ such that $M_{g} \in E_{r}\left(A_{g}\right)$. The remaining proof is same as ([5], Theorem $3.8)$, hence we omit it.

In the following result, (1) will follow from (2.3, 2.6) and (2) will follow from ([5, Lemma 3.10).

Lemma 3.3 Let $A=R\left[Y_{1}, \ldots, Y_{m}, f_{1}\left(l_{1}\right)^{-1}, \ldots, f_{n}\left(l_{n}\right)^{-1}\right]$ be a ring of the type $R[d, m, n]$ and $P$ a projective $A$-module of rank $r$. Then there exist an $s \in R, p_{1}, \ldots, p_{r} \in P$ and $\phi_{1}, \ldots, \phi_{r} \in$ $\operatorname{hom}(P, A)$ such that

(1) $P_{s}$ is free; $\left(\phi_{i}\left(p_{j}\right)\right)=$ diagonal $(s, \ldots, s) ; s P \subset p_{1} A+\ldots+p_{r} A$; the image of $s$ in $R_{\text {red }}$ is a non-zerodivisor; and $(0: s R)=\left(0: s^{2} R\right)$.

(2) Let $(a, p) \in \operatorname{Um}(A \oplus P, s A)$ with $p=c_{1} p_{1}+\ldots+c_{r} p_{r}$, where $c_{i} \in s A$ for all $i$. Assume there exist $\phi \in E_{r+1}^{1}(A, s)$ such that $\phi\left(a, c_{1}, \ldots, c_{r}\right)=(1,0, \ldots, 0)$. Then there exist $\Phi \in E(A \oplus P)$ such that $\Phi(a, p)=(1,0)$.

Following is the main result of this section which generalizes ([5], Theorem 3.12).

Theorem 3.4 Let $A=R\left[Y_{1}, \ldots, Y_{m}, f_{1}\left(l_{1}\right)^{-1}, \ldots, f_{n}\left(l_{n}\right)^{-1}\right]$ be a ring of type $R[d, m, n]$ and $P$ a projective $A$-module of rank $r \geq \max \{2, d+1\}$. Then $E(A \oplus P)$ acts transitively on $\operatorname{Um}(A \oplus P)$. In particular, $P$ is cancellative.

Proof Using ([5], Lemma 3.1), we may assume that $R$ is reduced. If $d=0$, then $R$ is a direct product of fields. Hence $P$ is free by (2.3) and the result follows from (3.1) with $s=1$. Assume $d>0$ and use induction on $d$.

By (3.3), there exist a non-zerodivisor $s \in R, p_{1}, \ldots, p_{r} \in P$ and $\phi_{1}, \ldots, \phi_{r} \in P^{*}$ satisfying the properties of $[3.3(1))$. We may assume that $s$ is not a unit, otherwise $P$ is free and we are done by (3.1). Rest of the proof is similar to ([5], Theorem 3.12) with $J=R$, we only give a sketch.

Let $(a, p) \in \mathrm{Um}(A \oplus P)$. Using (2.2) and induction on $d$, we may assume that $(a, p)=(1,0)$ modulo $s^{2} A$. By (3.3), $p=a_{1} p_{1}+\ldots+a_{r} p_{r}$ with $a_{i} \in s A$ and $\left(a, a_{1}, \ldots, a_{r}\right) \in \operatorname{Um}_{r+1}(A, s A)$. By (3.1), there exist $\phi \in E^{1}(A, s A)$ such that $\phi\left(a, a_{1}, \ldots, a_{r}\right)=(1,0, \ldots, 0)$. By $(3.3(2))$, we get $\Psi \in E(A \oplus P)$ such that $\Psi(a, p)=(1,0)$. This completes the proof.

Following result generalizes (2.10).

Proposition 3.5 Let $B$ be a reduced ring of dimension $d$ containing a field $k$ and $R$ an overring of $B[X]$ contained in its total quotient ring. Let $A=R\left[Y_{1}, \ldots, Y_{m}, f_{1}\left(l_{1}\right)^{-1}, \ldots, f_{n}\left(l_{n}\right)^{-1}\right]$ be a ring of type $R[\operatorname{dim} R, m, n]^{*}$ with $n \leq m, l_{i} \in k\left[Y_{1}, \ldots Y_{i}\right]$ and $a_{i i} \neq 0$. Then every projective A-module of rank $r \geq d+1$ is cancellative. 
Proof If $\operatorname{dim} R \leq d$ or $r \geq d+2$, then result follows from (3.4). Hence we assume $\operatorname{dim} R=d+1$ and $r=d+1$.

Step 1: We first prove that $A^{d+1}$ is cancellative. When $n=0$, we are done by (2.10). Assume $n>0$ and use induction on $n$.

Recall that $l_{n}=a_{n 1} Y_{1}+\ldots+a_{n n} Y_{n}-b_{n}$ with $a_{n n} \neq 0$. Changing $Y_{n} \mapsto a_{n n}^{-1}\left(Y_{n}-a_{n 1} Y_{1}-\right.$ $\left.\ldots-a_{n, n-1} Y_{n-1}\right)+b_{n}$, we can assume that $l_{n}=Y_{n}$. Let $P$ be a stably free $A$-module of rank $d+1$. If $S=1+f_{n}\left(Y_{n}\right) k\left[Y_{n}\right]$, then $\operatorname{dim} B\left[Y_{n}\right]_{f_{n}\left(Y_{n}\right) S}=d$. If $R^{\prime}=R\left[Y_{n}\right]_{f_{n} S}$, then $A_{S}=$ $R^{\prime}\left[Y_{1}, \ldots, Y_{n-1}, Y_{n+1}, \ldots, Y_{m}, f_{1}\left(l_{1}\right)^{-1}, \ldots, f_{n-1}\left(l_{n-1}\right)^{-1}\right]$ is a ring of type $R^{\prime}[d+1, m-1, n-1]^{*}$ with $l_{i} \in k\left[Y_{1}, \ldots Y_{i}\right]$ and $a_{i i} \neq 0$. By induction on $n, P_{S}$ is free. Hence we can find $g \in k\left[Y_{n}\right]$ such that $P_{1+f_{n} g}$ is free. If $C^{\prime}=R\left[Y_{1}, \ldots, Y_{n-1}, Y_{n+1}, \ldots, Y_{m}, f_{1}\left(l_{1}\right)^{-1}, \ldots, f_{n-1}\left(l_{n-1}\right)^{-1}\right]$ and $C=C^{\prime}\left[Y_{n}\right]$, then we have following fiber product diagram

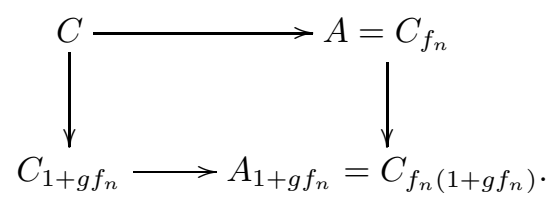

Since $P_{1+f_{n} g}$ is free, by (2.7), $P$ is extended from $C$, say $P_{f_{n}}^{\prime}=P$ for some projective $C$-module $P^{\prime}$. Since $P \oplus A \stackrel{\sim}{\rightarrow} A^{d+2}$, we get $\left(P^{\prime} \oplus C\right)_{f_{n}} \stackrel{\sim}{\rightarrow} C_{f_{n}}{ }^{d+2}$. Since $f_{n} \in C^{\prime}\left[Y_{n}\right]$ is a monic polynomial, using Suslin's monic inversion theorem $\left(\left[18\right.\right.$, Theorem 1), we get $P^{\prime} \oplus C \stackrel{\sim}{\rightarrow} C^{d+2}$. But $C$ is a ring of type $R[d+1, m, n-1]^{*}$ with $l_{i} \in k\left[Y_{1}, \ldots Y_{i}\right]$ and $a_{i i} \neq 0$. Hence by induction on $n, C^{d+1}$ is cancellative. Therefore, $P^{\prime}$ is free and so $P$ is free. This proves that $A^{r}$ is cancellative.

Step 2: We will prove the general case. Let $P$ be a projective $A$-module of rank $d+1$. If $d=0$, then we may assume that $B$ is a field. It is easy to see that $R=B\left[X, f(X)^{-1}\right]$ for some $f(X) \in B[X]$. Hence $A$ is a ring of type $B[0, m+1, n+1]$, so $P$ is free, by (2.3). Assume $d \geq 1$.

If $S$ is the set of non-zerodivisors of $B$, then as above, projective modules over $S^{-1} A$ are free. Hence we can choose $s \in S$ such that $P_{s}$ is free and $[3.3(1))$ holds. Note that if $B^{\prime}=B[T] /\left(T^{2}-\right.$ $\left.s^{2} T\right), R^{\prime}=R[T] /\left(T^{2}-s^{2} T\right)$ and $A^{\prime}=R^{\prime}\left[Y_{1}, \ldots, Y_{m}, f_{1}\left(l_{1}\right)^{-1}, \ldots, f_{n}\left(l_{n}\right)^{-1}\right]$, then $\left(A^{\prime}\right)^{d+1}$ is cancellative, by step 1 . By (2.9), every element of $\operatorname{Um}^{1}\left(A \oplus P, s^{2} A\right)$ can be taken to $(1,0)$ by some element of $A u t(A \oplus P)$. To complete the proof, it is enough to show that if $(a, p) \in \operatorname{Um}(A \oplus P)$, then there exist $\sigma \in A u t(A \oplus P)$ such that $\sigma(a, p) \in \mathrm{Um}^{1}\left(A \oplus P, s^{2} A\right)$.

Let "bar" denote reduction modulo $s^{2} A$. Then $\bar{A}=\bar{R}\left[Y_{1}, \ldots, Y_{m}, f_{1}\left(l_{1}\right)^{-1}, \ldots, f_{n}\left(l_{n}\right)^{-1}\right]$ and $\operatorname{dim} \bar{R} \leq d$. By (3.4), there exist $\bar{\sigma} \in E(\bar{A} \oplus \bar{P})$ such that $\bar{\sigma}(\bar{a}, \bar{p})=(1,0)$. Lifting $\bar{\sigma}$ to an element $\sigma \in E(A \oplus P)$, we get $\sigma(a, p) \in \mathrm{Um}^{1}\left(A \oplus P, s^{2} A\right)$. This completes the proof.

Remark 3.6 The result (3.5) is true for rings of type $R[d, m, n]$ such that $n \leq m, l_{i} \in k\left[Y_{1}, \ldots, Y_{i}\right]$, $a_{i i} \neq 0$ and each $f_{i}(T) \in R[T]$ is a monic polynomial. The proof is same as above by taking $S=1+f_{n}\left(Y_{n}\right) R\left[Y_{n}\right]$ and noting that $R^{\prime}$ is an overring of $R\left[Y_{n}\right]$. 


\section{Quillen's question and Bhatwadekar-Rao's results}

In this section we will generalize some results from [4] regarding Quillen's question mentioned in the introduction. We begin with the following:

Lemma 4.1 Let $R$ be a UFD of dimension 1 and $A=R\left[Y_{1}, \ldots, Y_{m}, f_{1}\left(l_{1}\right)^{-1}, \ldots, f_{n}\left(l_{n}\right)^{-1}\right]$ a ring of type $R[1, m, n]$. Then every projective A-module is free.

Proof If $n=0$, we are done by (4, Proposition 3.1). Assume $n>0$ and use induction on $n$. Let $P$ be a projective $A$-module of rank $r$. Using same arguments as in the proof of (3.1), after changing variables $\left(Y_{1}, \ldots, Y_{m}\right)$ by $\theta\left(Y_{1}, \ldots, Y_{m}\right)$ for some $\theta \in E_{m}(k)$, we may assume that $l_{n}=Y_{m}$.

Let $S=1+f_{n}\left(Y_{m}\right) R\left[Y_{m}\right]$ and $R^{\prime}=R\left[Y_{m}\right]_{f_{n} S}$. Then $R^{\prime}$ is a UFD of dimension 1 and $A_{S}=$ $R^{\prime}\left[Y_{1}, \ldots, Y_{m-1}, f_{1}\left(l_{1}\right)^{-1}, \ldots, f_{n-1}\left(l_{n-1}\right)^{-1}\right]$ is a ring of type $R^{\prime}[1, m-1, n-1]$, where $l_{i}=a_{i 1} Y_{1}+$ $\ldots+a_{i, m-1} Y_{m-1}-\widetilde{b}_{i}$ with $\widetilde{b}_{i}=b_{i}-a_{i m} Y_{m} \in R^{\prime}$ for $i=1, \ldots, n-1$. By induction on $n$, every projective $A_{S}$-module is free. In particular, $P_{S}$ is free. Find $1+f_{n} g \in S$ such that $P_{1+f_{n} g}$ is free. The ring $C=R\left[Y_{1}, \ldots, Y_{m}, f_{1}\left(l_{1}\right)^{-1}, \ldots, f_{n-1}\left(l_{n-1}\right)^{-1}\right]$ is of type $R[1, m, n-1]$. Hence by induction on $n$, projective $C$-modules are free. Consider the following fiber product diagram

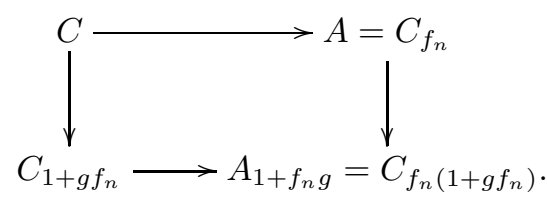

Since $P_{1+f_{n} g}$ is free, patching projective modules $P$ and $\left(C_{1+f_{n} g}\right)^{r}$ over $C_{f_{n}\left(1+f_{n} g\right)}$, we get that $P$ is extended from $C$ and hence $P$ is free.

\subsection{Infinite residue-field case}

The following result generalizes Bhatwadekar-Rao's Laurent polynomial case ([4], Theorem 3.2).

Proposition 4.2 Let $R$ be a regular $k$-spot of dimension $d$ with infinite residue field, $f$ a regular parameter of $R$ and $A=R\left[Y_{1}, \ldots, Y_{m}, f_{1}\left(l_{1}\right)^{-1}, \ldots, f_{n}\left(l_{n}\right)^{-1}\right]$ a ring of type $R[d, m, n]^{*}$. Then every projective $A_{f}$-module is free.

Proof Let $P$ be a projective $A_{f}$-module. If $T=R-\{0\}$, then $T^{-1} P$ is free, by (2.3). Find $g \in T$ such that $P_{g}$ is free. We may assume that $(g, f)$ is a regular sequence in $R$. By (2.8), there exist an infinite field $K / k$, a regular $K$-spot $R^{\prime}=K\left[Z_{1}, \ldots, Z_{d}\right]_{\left(\phi\left(Z_{1}\right), Z_{2}, \ldots, Z_{d}\right)}$ such that $R^{\prime} \subset R$ is an analytic isomorphism along $h \in g R \cap R^{\prime}$ and $f=Z_{d}$. Therefore, $A^{\prime}=R^{\prime}\left[Y_{1}, \ldots, Y_{m}, f_{1}\left(l_{1}\right)^{-1}, \ldots, f_{n}\left(l_{n}\right)^{-1}\right]$ is a ring of type $R^{\prime}[d, m, n]^{*}$ and $A^{\prime} \subset A$ is an analytic isomorphism along $h$. Since $P_{h}$ is free, by (2.7), $P$ is extended from $A_{Z_{d}}^{\prime}$. 
Enough to show that projective $A_{Z_{d}}^{\prime}$-modules are free. Replace $R^{\prime}$ by $R$ and $A^{\prime}$ by $A$. If $d \leq 2$, then $R_{Z_{d}}$ is a UFD of dimension $\leq 1$. Hence $P$ is free, by (4.1, 2.3). Assume $d>2$ and use induction on $d$. The proof is similar to (4], Theorem 3.2), hence we only give a sketch.

Let $S$ be multiplicative set of all non-zero homogeneous polynomials in $C=k\left[Z_{2}, \ldots, Z_{d}\right]$. Then $R_{Z_{d} S}$ is a localization of $C_{S}\left[Z_{1}\right]$. We can find $h \in C_{S}\left[Z_{1}\right]$ such that $P_{S}$ is defined over the ring $D=C_{S}\left[Z_{1}, h\left(Z_{1}\right)^{-1}, Y_{1}, \ldots, Y_{m}, f_{1}\left(l_{1}\right)^{-1}, \ldots, f_{n}\left(l_{n}\right)^{-1}\right]$. Note that $C_{S}$ is a UFD of dimension $\leq 1$, by (10, Proposition 1.11). Since $D$ is of type $C_{S}[1, m+1, n+1]$, by (4.1), $P_{S}$ is free. Choose $F \in S$ such that $P_{F}$ is free. Since $K$ is infinite, by linear change of variables, we can assume that $F$ is homogeneous and monic polynomial in $Z_{2}$ with coefficients in $k\left[Z_{3}, \ldots, Z_{d}\right]$.

If $\widetilde{R}=k\left[Z_{1}, Z_{3}, \ldots, Z_{d}\right]_{\left(\phi\left(Z_{1}\right), Z_{3}, \ldots, Z_{d}\right)}$, then $\widetilde{R}\left[Z_{2}\right] \subset R$ is an analytic isomorphism along $F$ (4], page 803). If $\widetilde{A}=\widetilde{R}\left[Z_{2}, Y_{1}, \ldots, Y_{m}, f_{1}\left(l_{1}\right)^{-1}, \ldots, f_{n}\left(l_{n}\right)^{-1}\right]$, then $\widetilde{A}_{Z_{d}} \subset A_{Z_{d}}$ is an analytic isomorphism along $F$. Since $P_{F}$ is free, $P$ is extended from $\widetilde{A}_{Z_{d}}$, by (2.7). Observe that $\widetilde{A}_{Z_{d}}$ is a ring of type $\widetilde{R}_{Z_{d}}[d-2, m+1, n]$. Hence by induction on $d$, projective $\widetilde{A}_{Z_{d}}$-modules are free. In particular, $P$ is free.

Recall that $R(T)$ denote the ring $S^{-1} R[T]$, where $S$ is the multiplicative set consisting of all monic polynomials of $R[T]$.

Corollary 4.3 Let $(R, \mathfrak{m})$ be a regular $k$-spot of dimension $d$ with infinite residue field. If $A=$ $R\left[Y_{1}, \ldots, Y_{m}, f_{1}\left(l_{1}\right)^{-1}, \ldots, f_{n}\left(l_{n}\right)^{-1}\right]$ is a ring of type $R[d, m, n]^{*}$, then projective modules over $A$ and $A \otimes_{R} R(T)$ are free.

Proof (i) Assume $P$ is a projective $A \otimes_{R} R(T)$-module. By (4, Corollary 3.5), $R(T)=R[X]_{(\mathfrak{m}, X)}[1 / X]$ with $X=T^{-1}$. Since $f=X \in R[X]_{(\mathfrak{m}, X)}$ is a regular parameter, we are done by (4.2).

(ii) Assume $P$ is a projective $A$-module. Then, we are done by $(i)$, using Suslin's monic inversion ([18, Theorem 1).

The laurent polynomial case of the following result is due to Popescu 14 .

Theorem 4.4 Let $(R, \mathfrak{m}, K)$ be a regular local ring of dimension d containing a field $k$ such that either char $k=0$ or char $k=p$ and tr-deg $K / \mathbb{F}_{p} \geq 1$. Let $f$ be a regular parameter of $R$ and $A=R\left[Y_{1}, \ldots, Y_{m}, f_{1}\left(l_{1}\right)^{-1}, \ldots, f_{n}\left(l_{n}\right)^{-1}\right]$ a ring of type $R[d, m, n]^{*}$. Then projective modules over $A, A_{f}$ and $A \otimes_{R} R(T)$ are free.

Proof ( $i$ ) Assume $P$ is a projective $A_{f}$-module. By (2.4), $R$ is a filtered inductive limit of some regular spots $\left(R_{i}\right)_{i \in I}$ over $\mathbb{Z}$, in particular over the prime subfield of $R$. Further, we may assume that $f$ is an extension of $f^{\prime} \in R_{j}$ for some $j$ and that $f^{\prime}$ is a regular parameter of $R_{j}$ (see [14).

Choosing possibly a bigger index $j \in I$, we may assume that $P$ is extended from $A_{f^{\prime}}^{\prime}$, where $A^{\prime}=R_{j}\left[Y_{1}, \ldots, Y_{m}, f_{1}\left(l_{1}\right)^{-1}, \ldots, f_{n}\left(l_{n}\right)^{-1}\right]$ is a ring of type $R_{j}[d, m, n]^{*}$. Since $\operatorname{tr}-\operatorname{deg} K / k \geq 1$, we can assume that the residue field of $R_{j}$ is infinite. By (4.2), $P^{\prime}$ and hence $P$ is free.

(ii) Following the proof of (4.3), projective modules over $A$ and $A \otimes_{R} R(T)$ are free. 


\subsection{Finite residue-field case}

The following result is an analogue of (4.2) in case residue field of $R$ is finite and generalizes Bhatwadekar-Rao's (4], Theorem 3.8).

Theorem 4.5 Let $R$ be a regular $\mathbb{F}_{q}$-spot of dimension $d, f$ a regular parameter of $R$ and $A=$ $R\left[Y_{1}, \ldots, Y_{m}, f_{1}\left(l_{1}\right)^{-1}, \ldots, f_{n}\left(l_{n}\right)^{-1}\right]$ a ring of type $R[d, m, n]^{*}$. Then every projective $A_{f}$-module of rank $\geq d-1$ is free.

Proof As in (4.2), using (2.8), we can assume $R=K\left[Z_{1}, \ldots, Z_{d}\right]_{\left(\phi\left(Z_{1}\right), Z_{2}, \ldots, Z_{d}\right)}$ and $f=Z_{d}$, where $K \supseteq \mathbb{F}_{q}$ may be a finite field. Let $P$ be a projective $A_{f}$-module of rank $r \geq d-1$. Note that projective $A_{f}$-modules are stably free and $\operatorname{dim} R_{f}=d-1$. Hence if $r \geq d$, then $P$ is free by 2.3 3.4). Therefore, we need to prove the result in case $r=d-1$. We use induction on $d$.

If $d \leq 2$, then $R_{f}$ is a UFD of dimension 1 and we are done by (4.1). Assume $d>2$. If $\widetilde{R}=K\left[Z_{1}, \ldots, Z_{d-1}\right]_{\left(\phi\left(Z_{1}\right), Z_{2}, \ldots, Z_{d-1}\right)}$, then $\widetilde{R}_{Z_{d-1}}$ is of dimension $d-2$. Since $R_{Z_{d} Z_{d-1}}$ is a localization of $\widetilde{R}_{Z_{d-1}}\left[Z_{d}\right]$, we can find $h\left(Z_{d}\right) \in \widetilde{R}_{Z_{d-1}}\left[Z_{d}\right]$ such that $P_{Z_{d-1}}$ is defined over $C=$ $\widetilde{R}_{Z_{d-1}}\left[Z_{d}, h\left(Z_{d}\right)^{-1}, Y_{1}, \ldots, Y_{m}, f_{1}\left(l_{1}\right)^{-1}, \ldots, f_{n}\left(l_{n}\right)^{-1}\right]$. Since $C$ is of type $\widetilde{R}_{Z_{d-1}}[d-2, m+1, n+1]$ by (3.4), $P_{Z_{d-1}}$ being stably free is free.

If $R^{\prime}=K\left[Z_{1}, \ldots, Z_{d-2}, Z_{d}\right]_{\left(\phi\left(Z_{1}\right), Z_{2}, \ldots, Z_{d-2}, Z_{d}\right)}$, then $R_{Z_{d}}^{\prime}\left[Z_{d-1}\right] \subset R_{Z_{d}}$ is an analytic isomorphism along $Z_{d-1}$ (see [4], page 803). If $A^{\prime}=R_{Z_{d}}^{\prime}\left[Z_{d-1}, Y_{1}, \ldots, Y_{m}, f_{1}\left(l_{1}\right)^{-1}, \ldots, f_{n}\left(l_{n}\right)^{-1}\right]$ then $A_{Z_{d}}^{\prime} \subset A_{Z_{d}}$ is also an analytic isomorphism along $Z_{d-1}$. Using $P_{Z_{d-1}}$ is free, $P$ is extended from $D=R_{Z_{d}}^{\prime}\left[Z_{d-1}, Y_{1}, \ldots, Y_{m}, f_{1}\left(l_{1}\right)^{-1}, \ldots, f_{n}\left(l_{n}\right)^{-1}\right]$. By induction on $d$, projective $D$-modules of rank $\geq d-1$ are free. Hence $P$ is free.

The following result is an analogue of (4.3) in case residue field of $R$ is finite and follows from (4.5) by following the proof of (4.3).

Corollary 4.6 Let $R$ be a regular $\mathbb{F}_{q}$-spot of dimension $d$, and $A$ a ring of type $R[d, m, n]^{*}$. Then projective modules of rank $\geq d$ over $A$ and $A \otimes_{R} R(T)$ are free.

The proof of following result is exactly same as (4, Proposition 4.1, Theorem 4.2) using (2.3). Hence we omit the proof.

Theorem 4.7 Let $R=\mathbb{F}_{p}\left[\left[Z_{1}, \ldots, Z_{d}\right]\right]$ and $f$ be a regular parameter of $R$. If $A$ is a ring of type $R[d, m, n]^{*}$, then projective modules over $A, A_{f}, A \otimes_{R} R(T)$ are free.

\section{Generalization of Rao's results}

In this section, we will generalize some results from [17]. We begin with the following result. It's proof is exactly same as ([17, Theorem 2.1) by using (3.4) instead of Swan's result, hence we omit it. The case $t \leq 1$ is (4.7). 
Proposition 5.1 Let $(R, \mathfrak{m})$ be a formal (or convergent) power series ring of dimension $d$ over a field $k$. Let $g_{1}, \ldots, g_{t}$ be regular parameters of $R$ which are linearly independent modulo $\mathfrak{m}^{2}$. If $A$ is a ring of type $R[d, m, n]^{*}$, then every projective $A_{g_{1} \ldots g_{t}}$-module of rank $\geq t-1$ is free.

Lemma 5.2 Let $(R, \mathfrak{m})$ be a regular $k$-spot of dimension $d$ and $S$ a multiplicative closed subset of $R$ which contains a regular parameter of $R$. Let $A=R\left[Y_{1}, \ldots, Y_{m}, f_{1}\left(l_{1}\right)^{-1}, \ldots, f_{n}\left(l_{n}\right)^{-1}\right]$ be a ring of type $R[d, m, n]^{*}$. Then every projective $S^{-1} A$-module of rank $\geq d-1$ is free.

Proof Since $\operatorname{dim} S^{-1} R \leq d-1$, if rank $P>d-1$, then we are done by (3.4). Assume that rank $P=d-1$. We will follow the notation and proof of ([17, Proposition 2.3). If we show that every stably free module $P$ of rank $d-1$ over $R_{Z_{1} s}^{\prime}\left[Y_{1}, \ldots, Y_{m}, f_{1}\left(l_{1}\right)^{-1}, \ldots, f_{n}\left(l_{n}\right)^{-1}\right]$ is free, then remaining proof is exactly same as in [17.

Recall that $R^{\prime}=K\left[Z_{1}, \ldots, Z_{d}\right]_{\left(Z_{1}, \ldots, Z_{d-1}, \phi\left(Z_{d}\right)\right)}$. If $\widetilde{R}=K\left[Z_{1}, \ldots, Z_{d-2}, Z_{d}\right]_{\left(Z_{1}, \ldots, Z_{d-2}, \phi\left(Z_{d}\right)\right)}$, then $R_{Z_{1} s}^{\prime}$ is a localization of $\widetilde{R}_{Z_{1} s}\left[Z_{d-1}\right]$. We can find $f\left(Z_{d-1}\right) \in \widetilde{R}_{Z_{1} s}\left[Z_{d-1}\right]$ such that $P$ is defined over $C=\widetilde{R}_{s Z_{1}}\left[Z_{d-1}, f^{-1}, Y_{1}, \ldots, Y_{m}, f_{1}\left(l_{1}\right)^{-1}, \ldots, f_{n}\left(l_{n}\right)^{-1}\right]$. Since $C$ is a ring of type $\widetilde{R}_{Z_{1}}[d-2, m+1, n+1]$ and $P$ is stably free of rank $d-1$, by (3.4), $P$ is free. This completes the proof.

The following result is immediate from (5.2).

Corollary 5.3 Let $(R, \mathfrak{m})$ be a regular $k$-spot of dimension 3 and $f, g, h$ regular parameters of $R$ which are linearly independent modulo $\mathfrak{m}^{2}$. Let $A$ be a ring of type $R[3, m, n]^{*}$. Then projective modules over $A, A_{f}, A_{f g}$ and $A_{f g h}$ are free.

Lemma 5.4 Let $k$ be an infinite field, $B=k\left[Z_{1}, \ldots, Z_{d}\right], \mathfrak{m}=\left(Z_{1}, \ldots, Z_{d-1}, \phi\left(Z_{d}\right)\right)$ a maximal ideal and $R=B_{\mathfrak{m}}$. Let $A=R\left[Y_{1}, \ldots, Y_{m}, f_{1}\left(l_{1}\right)^{-1}, \ldots, f_{n}\left(l_{n}\right)^{-1}\right]$ be a ring of type $R[d, m, n]^{*}$ and $h \in k\left[Z_{1}, \ldots, Z_{t}\right]$. Then every projective $A_{h}$-module of rank $\geq t$ is free.

Proof Assume $t=d$. If $h \in \mathfrak{m}$, then $\operatorname{dim} R_{h}=d-1$ and the result follows from (3.4). If $h \notin \mathfrak{m}$, then $R_{h}=R$ and we are done by (4.3). The proof in case $t<d$ is similar to ([17], Proposition $2.7)$, hence we only give a sketch.

We can find $f \in B-\mathfrak{m}$ such that $P$ is defined over $B\left[(f h)^{-1}, Y_{1}, \ldots, Y_{m}, f_{1}\left(l_{1}\right)^{-1}, \ldots, f_{n}\left(l_{n}\right)^{-1}\right]$. If $S=k\left[Z_{t+1}, \ldots, Z_{d}\right]-(0)$, then $P_{S}$ is defined over $\widetilde{R}\left[Y_{1}, \ldots, Y_{m}, f_{1}\left(l_{1}\right)^{-1}, \ldots, f_{n}\left(l_{n}\right)^{-1}\right]$, where $\widetilde{R}=K\left[Z_{1}, \ldots, Z_{t}\right] \mathfrak{m}_{1}\left[(f h)^{-1}\right]$ with $K=k\left(Z_{t+1}, \ldots, Z_{d}\right)$ and $\mathfrak{m}_{1}=\left(Z_{1}, \ldots, Z_{t}\right)$. Since rank $P \geq t$ and $\operatorname{dim} \widetilde{R} \leq t, P_{S}$ is free $(t=d$ case).

Proceed as in [17, we get that if $B^{\prime}=k\left[Z_{1}, \ldots, Z_{d-1}\right]_{\left(Z_{1}, \ldots, Z_{d-1}\right)}$, then $P$ is extended from $C_{h}$, where $C=B^{\prime}\left[Z_{d}, Y_{1}, \ldots, Y_{m}, f_{1}\left(l_{1}\right)^{-1}, \ldots, f_{n}\left(l_{n}\right)^{-1}\right]$. Since $C_{h}$ is of type $B_{h}^{\prime}\left[d^{\prime}, m+1, n\right]^{*}$, where $d^{\prime} \leq d-1$, by induction on $d, P$ is free.

Lemma 5.5 Let $K$ be an infinite field and $R=K\left[Z_{1}, \ldots, Z_{d}\right] \mathfrak{m}$, where $\mathfrak{m}=\left(Z_{1}, \ldots, Z_{d-1}, \phi\left(Z_{d}\right)\right)$ is a maximal ideal. Fix $q>0$ an integer such that $d \geq 2 q-1$. Let $B=R_{h g_{1} \ldots g_{k}}$, where $h \in$ $K\left[Z_{1}, \ldots, Z_{p}\right]$ with $1 \leq p<q$ and $g_{1}, \ldots, g_{k}$ are regular parameters of $\mathfrak{m}$ with $Z_{1}, \ldots, Z_{p}, g_{1}, \ldots, g_{k}$ 
linearly independent modulo $\mathfrak{m}^{2}$. Let $A=B\left[Y_{1}, \ldots, Y_{m}, f_{1}\left(l_{1}\right)^{-1}, \ldots, f_{n}\left(l_{n}\right)^{-1}\right]$ be a ring of type $B[d-1, m, n]^{*}$ and $P$ a projective $A$-module of rank $\geq d-q$. Then there exist $g \in k\left[Z_{1}, \ldots, Z_{p}\right]$ such that $P_{g}$ is free.

Proof We follow the proof and notations of ([17, Proposition 2.8) and indicate the necessary changes. If $k=0$, then $B=R_{h}$ with $h \in K\left[Z_{1}, \ldots, Z_{p}\right]$. In this case, using (5.4), $P$ itself is free. Assume $k>0$ and use induction on $k$. Proceed as in [17] using (5.2). Let $S=K\left[Z_{1}, \ldots, Z_{q}\right]-(0)$. We only need to show that $S^{-1} P$ is free. Remaining arguments are same as in [17.

Recall that $g_{1}=Z_{p+1}, \ldots, g_{q-p}=Z_{q}$. Write $\widetilde{R}=K\left(Z_{1}, \ldots, Z_{q}\right)\left[Z_{q+1}, \ldots, Z_{d}\right]_{\mathfrak{m}^{\prime}}$, where $\mathfrak{m}^{\prime}=$ $\left(Z_{q+1}, \ldots, Z_{d-1}, \phi\left(Z_{d}\right)\right)$. Then $S^{-1} P$ is defined over $C=\widetilde{R}_{g_{q-p+1} \ldots g_{k}}\left[Y_{1}, \ldots, Y_{m}, f_{1}\left(l_{1}\right)^{-1}, \ldots, f_{n}\left(l_{n}\right)^{-1}\right]$.

Assume $k<q-p+1$. Then $C=\widetilde{R}\left[Y_{1}, \ldots, Y_{m}, f_{1}\left(l_{1}\right)^{-1}, \ldots, f_{n}\left(l_{n}\right)^{-1}\right]$ and $S^{-1} P$ is free, by (4.3). If $k \geq q-p+1$, use (5.2) to conclude that $S^{-1} P$ is free.

Theorem 5.6 Let $(R, \mathfrak{m})$ be a regular $k$-spot of dimension d with infinite residue field. Let $A=$ $R_{g_{1} \ldots g_{t}}\left[Y_{1}, \ldots, Y_{m}, f_{1}\left(l_{1}\right)^{-1}, \ldots, f_{n}\left(l_{n}\right)^{-1}\right]$ be a ring of type $R_{g_{1} \ldots g_{t}}[d-1, m, n]^{*}$, where $g_{1}, \ldots, g_{t}$ are regular parameters of $R$ which are linearly independent modulo $\mathfrak{m}^{2}$. Then every projective $A$-module $P$ of rank $r \geq \min \{t,[d / 2]\}$ is free.

Proof We will follow the proof and notations of (17, Theorem 2.9). If we show that $S^{-1} P$ is free, then rest of the argument is same as in [17. Note $R^{\prime}=k\left[Z_{1}, \ldots, Z_{d}\right]_{\left(Z_{1}, \ldots, Z_{d-1}, \phi\left(Z_{d}\right)\right)}$ and $P$ is defined over $R_{Z_{1} \ldots Z_{t}}^{\prime}\left[Y_{1}, \ldots, Y_{m}, f_{1}\left(l_{1}\right)^{-1}, \ldots, f_{n}\left(l_{n}\right)^{-1}\right]$. Write $S=k\left[Z_{1}, \ldots, Z_{q}\right]-(0)$ and $\widetilde{R}=$ $K\left(Z_{1}, \ldots, Z_{q}\right)\left[Z_{q+1}, \ldots, Z_{d}\right]_{\left(Z_{q+1}, \ldots, Z_{d-1}, \phi\left(Z_{d}\right)\right)}$. Then $R^{\prime}$ is a localization of $\widetilde{R}$. We can find $h_{1} \in$ $K\left[Z_{1}, \ldots, Z_{d}\right]$ such that $S^{-1} P$ is defined over $C=\widetilde{R}_{h_{1} Z_{q+1} \ldots Z_{t}}\left[Y_{1}, \ldots, Y_{m}, f_{1}\left(l_{1}\right)^{-1}, \ldots, f_{n}\left(l_{n}\right)^{-1}\right]$. Since $\operatorname{dim} \widetilde{R}=d-q$ which is $q$ if $d$ is even and $q+1$ when $d$ is odd, $S^{-1} P$ is free, by (5.2).

The following result is an analog of (5.1) for regular $k$-spots in the geometric case. Recall that a local ring $(R, \mathfrak{m})$ is said to have a coefficient field if $R$ contains a subfield $K$ isomorphic to $R / \mathfrak{m}$. The proof is exactly same as of ([17, Theorem 2.12) using above results. Hence we omit the proof.

Theorem 5.7 Let $(R, \mathfrak{m})$ be a regular $k$-spot with infinite residue field. Let $g_{1}, \ldots, g_{t}$ be regular parameters of $R$ which are linearly independent modulo $\mathfrak{m}^{2}$. Assume that $R /\left(g_{1}\right)$ contains a coefficient field. If $A=R_{g_{1} \ldots g_{t}}\left[Y_{1}, \ldots, Y_{m}, f_{1}\left(l_{1}\right)^{-1}, \ldots, f_{n}\left(l_{n}\right)^{-1}\right]$ is of type $R_{g_{1} \ldots g_{t}}[d-1, m, n]^{*}$, then every projective $A$-module $P$ of rank $\geq t-1$ is free.

The following result generalizes Popescu's result [14. For $t \leq 1$, this follows from (4.4).

Theorem 5.8 Let $(R, \mathfrak{m}, K)$ be a regular local ring of dimension d containing a field $k$ such that either char $k=0$ or char $k=p$ and tr-deg $K / \mathbb{F}_{p} \geq 1$. Let $g_{1}, \ldots, g_{t}$ be regular parameters of $R$ which are linearly independent modulo $\mathfrak{m}^{2}$. Let $A=R_{g_{1} \ldots g_{t}}\left[Y_{1}, \ldots, Y_{m}, f_{1}\left(l_{1}\right)^{-1}, \ldots, f_{n}\left(l_{n}\right)^{-1}\right]$ be a ring of type $R_{g_{1} \ldots g_{t}}[d-1, m, n]^{*}$. Then every projective $A$-module of rank $\geq t$ is free. 
Proof We follow the proof of (4.4) and use same notations. As in [14], if $g=g_{1} \ldots g_{t}$, then $g$ is an extension of $g^{\prime} \in R_{j}$ for some $j$. Further, $g^{\prime}$ is a product of regular parameters $g_{1}^{\prime}, \ldots, g_{t}^{\prime}$ of $\left(R_{j}, \mathfrak{m}_{j}\right)$ which are linearly independent modulo $\mathfrak{m}_{j}^{2}$. If $P$ is a projective $A_{f}$-module, then by choosing possibly a bigger index $j \in I$, we may assume that $P$ is an extension of a projective module $P^{\prime}$ over $A^{\prime}$, where $A^{\prime}=\left(R_{j}\right)_{g^{\prime}}\left[Y_{1}, \ldots, Y_{m}, f_{1}\left(l_{1}\right)^{-1}, \ldots, f_{n}\left(l_{n}\right)^{-1}\right]$. If $\operatorname{dim} R^{\prime}=d^{\prime}$, then $A^{\prime}$ is a ring of type $\left(R_{j}\right)_{g^{\prime}}\left[d^{\prime}-1, m, n\right]^{*}$. Now $R_{j}$ is a regular $k$-spot. Since tr-deg $K / k \geq 1$, we can assume that the residue field of $R_{j}$ is infinite. By (5.6),$P^{\prime}$ and hence $P$ is free.

The following result is immediate from (5.8).

Corollary 5.9 Let $(R, \mathfrak{m}, K)$ be a regular local ring of dimension d containing a field $k$ such that either char $k=0$ or char $k=p$ and tr-deg $K / \mathbb{F}_{p} \geq 1$. Let $f, g$ be regular parameters of $R$ which are linearly independent modulo $\mathrm{m}^{2}$. If $A$ is a ring of type $R[d, m, n]^{*}$, then every projective module over $A, A_{f}$ and $A_{f g}$ are free.

Acknowledgements: We would like to thank Professor D. Popescu for sending us a copy of 14 and Mrinal K. Das for bringing Gabber's result to our notice.

\section{References}

[1] A. Bak, R. Basu and R.A. Rao, Local-global principle for transvection groups, Proc. Amer. Math. Soc. 138 (2010) 1191-1204.

[2] H. Bass, K-theory and stable algebra, IHES 22 (1964) 5-60.

[3] H. Bass, Some problems in classical algebraic K-Theory II, Lect. Not. Math. 342, Springer Verlag (1972).

[4] S.M. Bhatwadekar and R.A. Rao, On a question of Quillen, Trans. Amer. Math. Soc. 279 (1983) $801-810$.

[5] A.M. Dhorajiya and M.K. Keshari, Projective modules over overrings of polynomial rings, J. Algebra 323 (2010) 551-559.

[6] O. Gabber, On purity theorems for vector bundles, Int. Math. Res. Not. 15 (2002) 783-788

[7] M.K. Keshari, Cancellation problem for projective modules over affine algebras, J. K-Theory 3 (2009) $561-581$.

[8] H. Lindel, Unimodular elements in projective modules, J. Algebra 172 (1995) 301-319.

[9] H. Lindel, On a question of Bass, Quillen and Suslin concerning projective modules over polynomial rings, Invent. Math. 65 (1981) 319-323.

[10] B.S. Nashier, Efficient generation of ideals in polynomial rings, J. Algebra 85 (1983) 287-302.

[11] B.S. Nashier, On the conormal bundle of ideals, J. Algebra 85 (1983) 361-367.

[12] B. Plumstead, The conjectures of Eisenbud and Evans, Amer. J. Math. 105 (1983) 1417-1433.

[13] D. Popescu, Polynomial rings and their projective modules, Nagoya Math J. 113 (1989) 121-128. 
[14] D. Popescu, On a question of Quillen, Bull. Math. Soc. Sci. Math. Roumanie (N.S.) 45 (93) no. 3-4 (2002) 209212.

[15] D. Quillen, Projective modules over polynomial rings, Invent. Math. 36 (1976) 167-171.

[16] R.A. Rao, A question of H. Bass on the cancellative nature of large projective modules over polynomial rings, Amer. J. Math. 110 (1988) 641-657.

[17] R.A. Rao, On projective $R_{f_{1} \ldots f_{t}}$-modules, Amer. J. Math. 107 (1985) 387-406.

[18] A.A. Suslin, Projective modules over a polynomial ring are free, Soviet Math. Dokl. 17 (1976) 11601164.

[19] R.G. Swan, Gubeladze's proof of Anderson's conjecture, Azumaya algebras, actions and modules, Contemp. Math. 124 (1992) 215-250.

[20] A. Wiemers, Cancellation properties of projective modules over Laurent polynomial rings, J. Algebra, 156 (1993) 108-124. 